\title{
RADAR OBSERVATIONS OF THE MOON AT Io-CM WAVELENGTH
}

\author{
J. S. HEY AND V. A. HUGHES \\ Royal Radar Establishment, Malvern, England
}

This note describes some of the initial results derived from observations of radar echoes from the moon obtained at $10-\mathrm{cm}$ wavelength at the Royal Radar Establishment, Malvern. The radar, which has been described elsewhere [1], has a transmitter of 2 megawatts peak power with a pulse length of 5 microseconds and a pulse recurrence frequency of 260 per second. The receiver is of conventional design and has a bandwidth of $500 \mathrm{kc} / \mathrm{s}$ and a noise factor of 7.5. The aerial used is the 45 -foot diameter radio telescope shown in Fig. 1. The telescope is controlled from a mechanical computer that converts the lunar coordinates into azimuth and elevation, which are then fed into a servo drive.

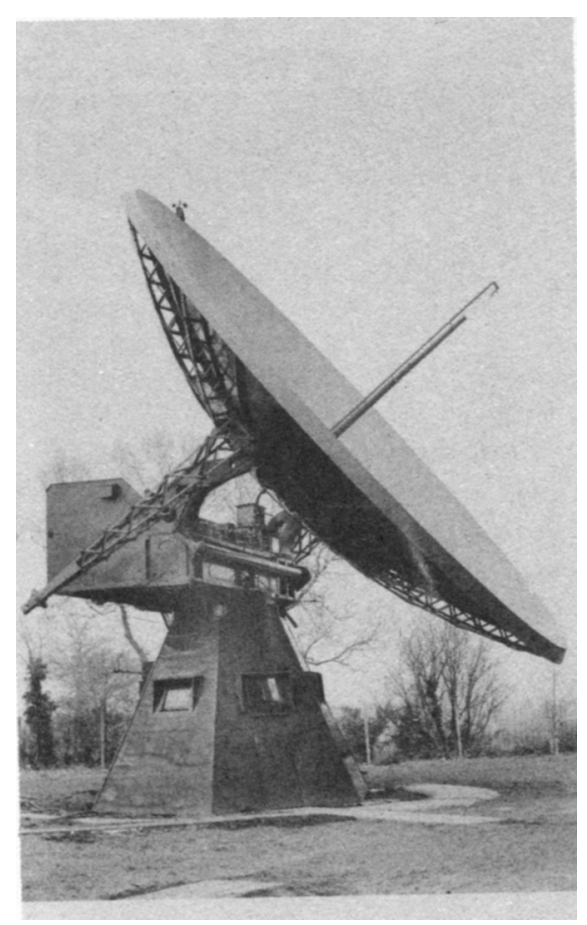

FIg. 1. The radio telescope.
The moon echoes were observed on two types of display: on one the signal was displayed as' a deflection of a time base on a cathode-ray tube, and on the other as a brightness modulation of the trace. Recordings were made by photographing the cathoderay tube with the brightness-modulated time base, which was made to move slowly across the tube. Each frame then showed a moon echo with coordinates of range and time and with echo amplitude appearing as a variation in density on the film. An example of the type of record is shown in Fig. 2. The echo may be examined in more detail by using a recording photometer. Two types of analysis were then made: a study of the shape of the echo in range, and measurements on the rate of fluctuation at different distances from the leading edge.

A main characteristic of the echo is the initial sharp rise in intensity and 
the rapid falling off with range. This is illustrated in Fig. 3, obtained from a photometer record. The record appears to consist of a series of random pulses, of length equal to the transmitter pulse length, with the mean amplitude disappearing into the receiver noise in a distance of about $50 \mathrm{~km}$. When the variation in echo amplitude is compared with the angle of incidence at the moon's surface, it is found that the mean level is reduced to about 50 per cent of the initial value at an angle of about 5 degrees, thus indicating a highly specular reflecting surface. That the surface consists of a large number of scatterers is indicated by Fig. 4 , in which the number of peaks at different distances from the leading edge is plotted for eleven records taken at intervals of two minutes.

The radar range to the moon may be calculated by using records of the type shown in Fig. 3. Since the time interval between transmitter pulses corresponds to a distance of about $550 \mathrm{~km}$, the distance between the radar and the front surface of the moon must first be calculated from tabulated values of lunar horizontal parallax corrected for the effect of the radar geocentric distance and the radius of the moon. From an accurate measurement

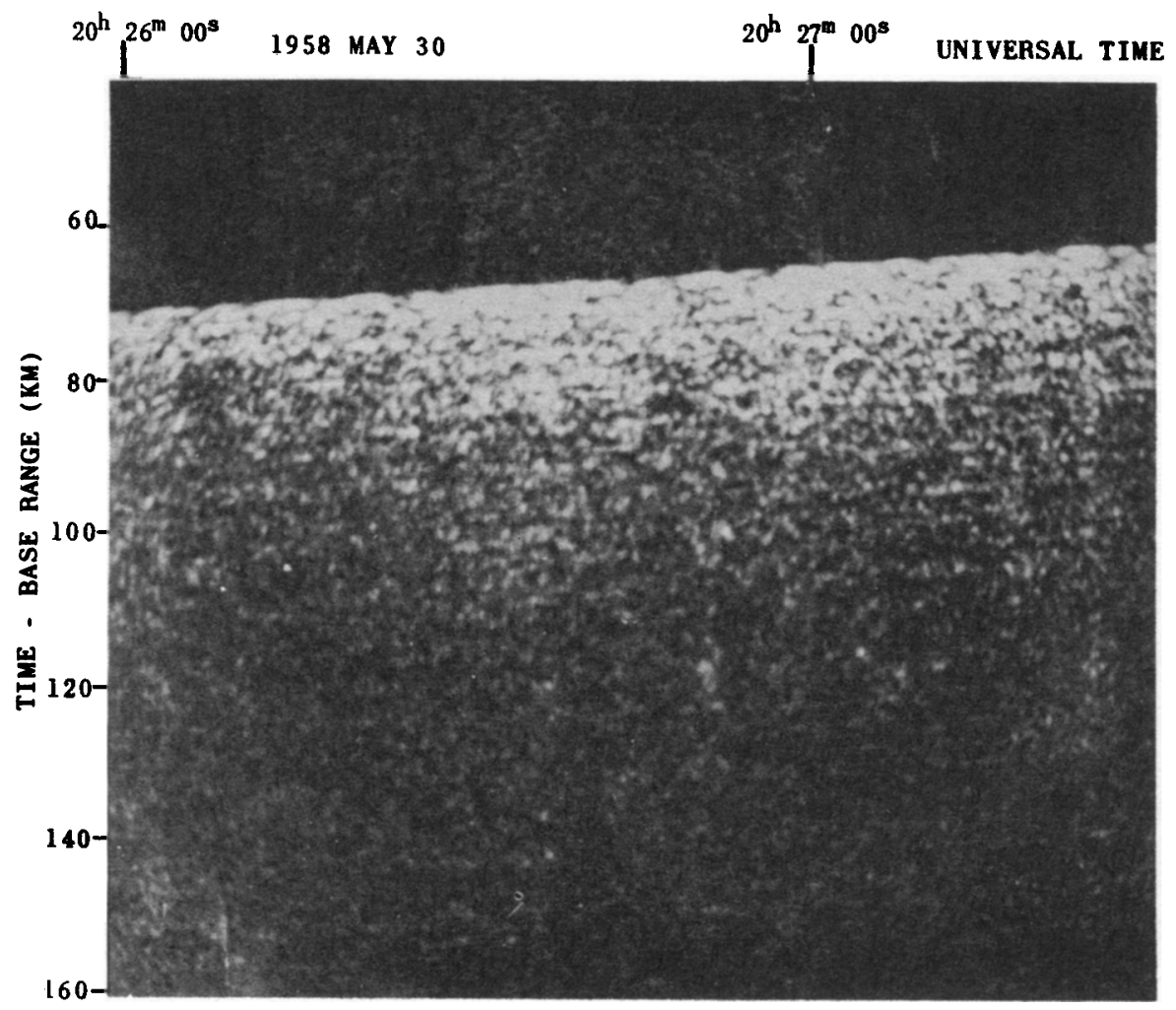

Fig. 2. Moon echo on range-time displays. 
of the time interval between transmitter pulses the time-base range at which the moon echo is expected to appear may be determined, and compared with that obtained from the records. Fig. 5 shows the variation in time-base range to the moon near transit on 1958 May 30, and Fig. 6 shows the difference between the radar and calculated ranges. In the calculations the velocity of propagation is assumed to be $299,791.0$ $\mathrm{km} / \mathrm{second}$, the equatorial radius of the earth $6378.388 \mathrm{~km}$, and the geo-

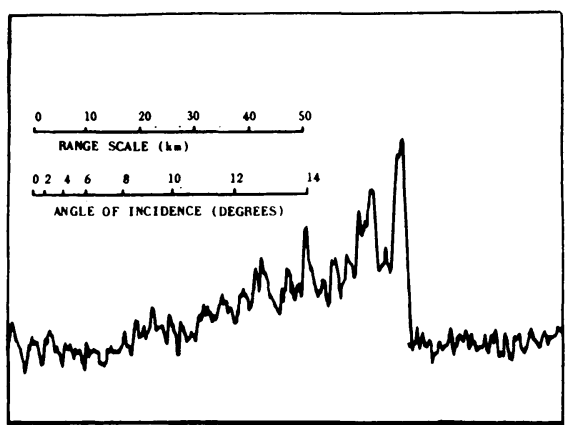

Fig. 3. Example of moon echo obtained with recording photometer.

centric distance $6365.0 \mathrm{~km}$ at latitude $52^{\circ} \mathrm{N}$. The values for the moon's horizontal parallax have been taken from the Improved Lunar Ephemeris (1952-59). The over-all agreement in range is good, and the mean difference of $1.53 \mathrm{~km}$ with a root-mean-square deviation of $1.25 \mathrm{~km}$ is comparable to similar measurements by Yaplee et al. [2]. Most of the scatter in range is likely to be due to variation in amplitude of the signal at the leading edge. No correction has been made for atmospheric refraction, and it is assumed that the changes in velocity of propagation caused by any departures of the refractive index from the value in vacuo are comparatively small.

From the decrease in amplitude of the signal with range it would appear that the surface of the moon scatters over a mean angle of about 5 degrees. This may be explained by assuming that the surface of the moon producing the echo consists mainly of almost flat regions presumably covered by dust, and that the mountains and craters occupy only a small percentage of the

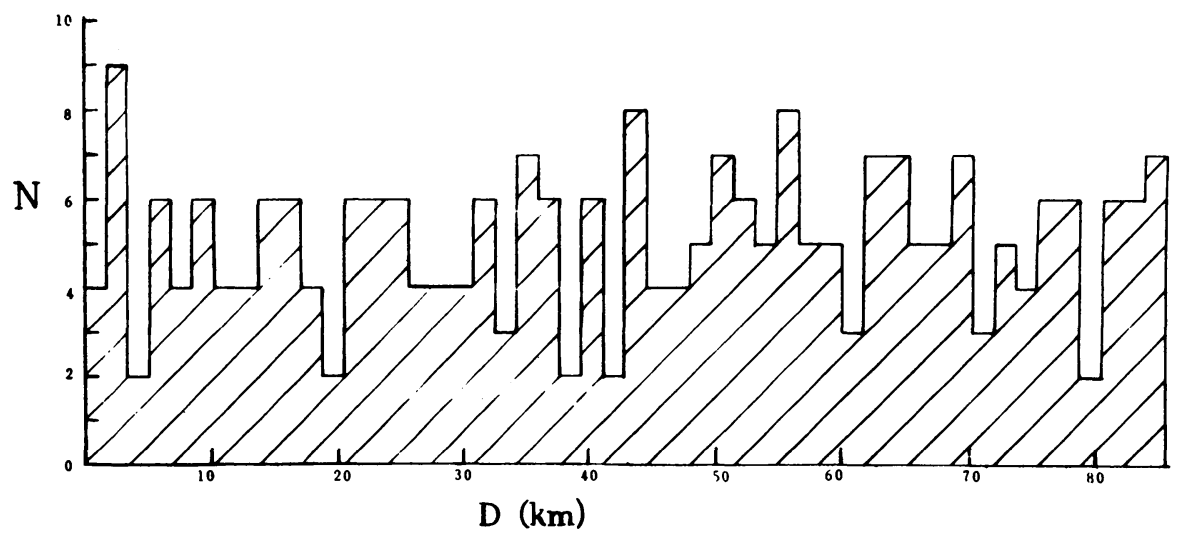

Fig. 4. Number of peaks $(N)$ at distance $(D)$ from leading edge of moon echo, taken over 11 records, 1958 May $24,17^{\mathrm{h}} 5^{\mathrm{m}}$ to $18^{\mathrm{h}} 14^{\mathrm{m}}$ U.T. 


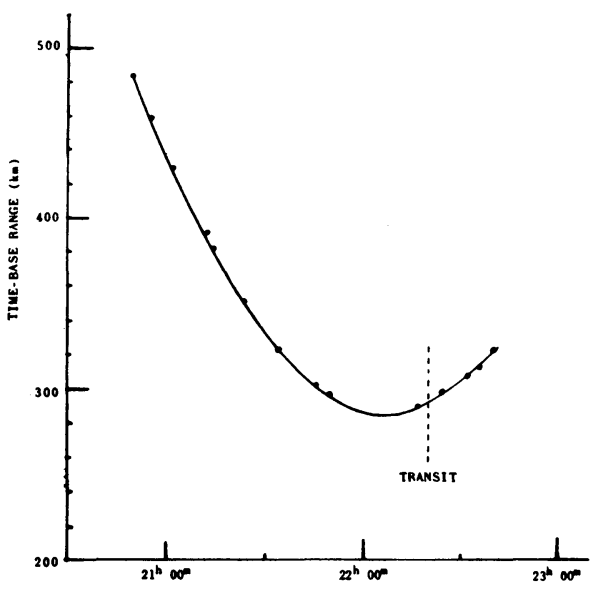

FIG. 5. Radar range to moon during transit, 1958 May 30.

total area. Under these conditions we may assume that the reflection coefficient at the surface is constant, and hence that the reflected wave will consist of a wave of nearly uniform amplitude (since the regions are close to normal incidence) but with phase modulation determined by twice the amplitude of the undulations in the lunar surface. Also since the received echo originates from regions greater in area than that of the first Fresnel zone, the received radiation will consist of a wave with both amplitude and phase modulation, and may be considered analogous to the case of radio star scintillation produced by ionospheric diffraction. By arguments similar to those developed by Hewish [3] for the case of the ionosphere, the value of 5 degrees for the scattering angle of the reflected wave would be consistent with a mean scale for the phase modulation at the lunar surface of about 1 meter. However, since the amplitude modulation exceeds 100 per cent, and this is supported by observations on A-scope displays, the phase modulation is likely to be greater than $2 \pi$. Hence it may be concluded that the lunar topography is such that the reflected wave is changed in phase by $2 \pi$ in a distance of about 1 meter; i.e., the mean gradient of the lunar surface is about 1 in 20.

Under these conditions we may expect that the scintillation rate at the leading edge of the moon echo would be related to the phase scale and to the lunar velocity normal to the line of sight produced by libration and would correspond to fluctuations of intensity with a period of a few seconds. At distances behind the leading edge where the angle of incidence is greater, the fluctuation rate would be expected to be more rapid owing to the increase

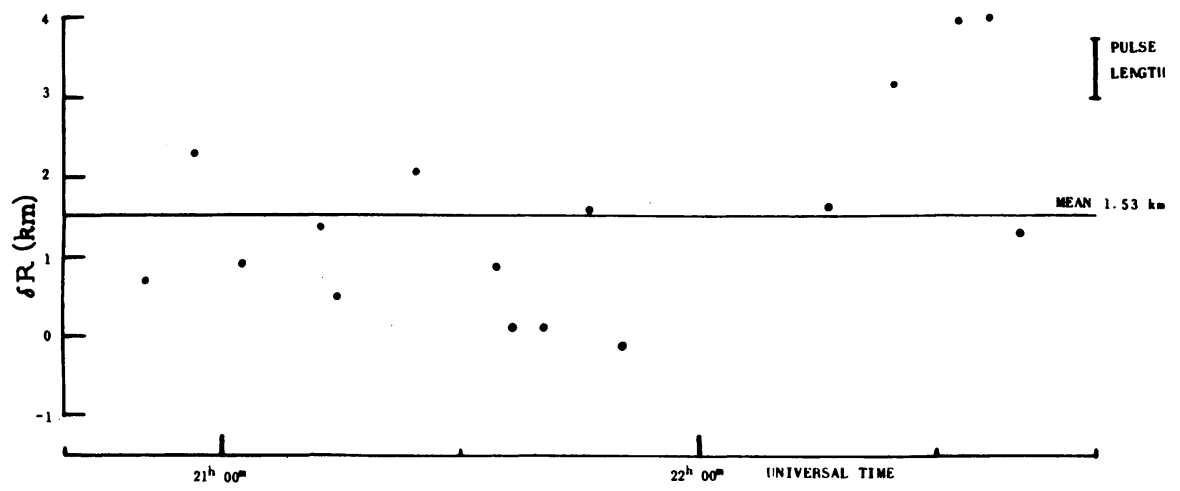

FIG. 6. Difference between radar and computed range to moon $(\delta R), 1958$ May 30. 


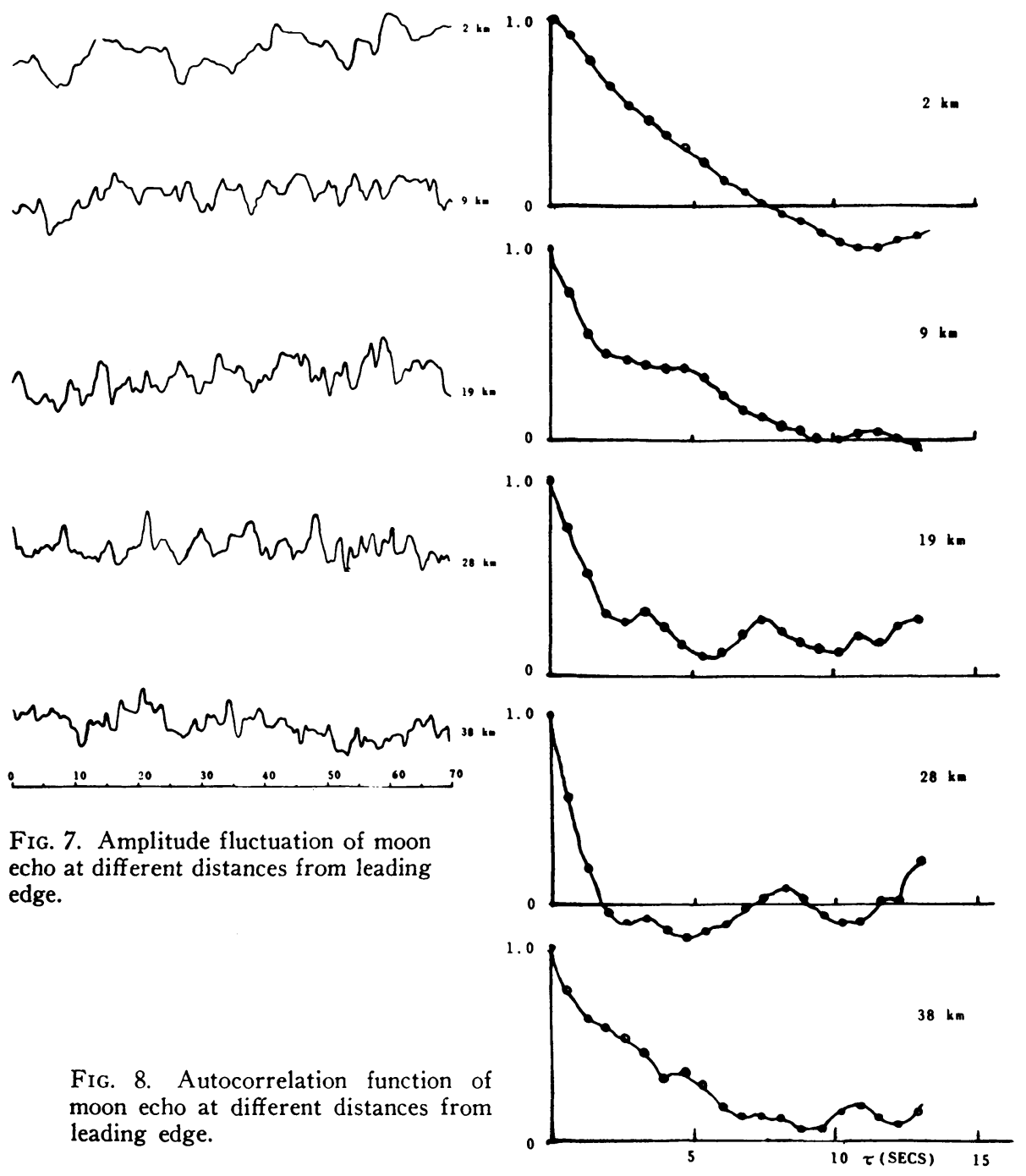

in total phase path and to the more rapid rate of change of lunar topography. The increase in frequency is shown in Fig. 7 and illustrated in Fig. 8 by means of the autocorrelation functions, which show an increased periodicity with distance behind the leading edge. In Figs. 7 and 8 some of the highfrequency components are reduced owing to the method of analysis, and the low-frequency components, which may be produced by receiver instability, are enhanced.

In conclusion it may be stated that these results are preliminary, but may provide a useful model to explain some of the characteristics of moon echoes.

Acknowledgement is made to $\mathrm{Mr}$. D. Barber for his assistance in the observations and in the analysis of the results. 


\section{REFERENCES}

[1] Staff of the Royal Radar Establishment. Nature, 180, 1225, 1957.

[2] Yaplee, B. S., Bruton, R. H., Craig, K. J., and Roman, N. G. Proc. I.R.E. 46, 293, 1958.

[ 3] Hewish, A. Proc. Roy. Soc. 209, 81, 1951; 214, 494, 1952.

\section{Discussion}

Gold: I think it may be instructive to compare the moon radar results with those obtained by airborne radar on the earth, to see what terrain produces similar results. We should be able to compare the power scattered at different angles to the surface and calculate what length of echo this would produce on the moon. Mountainous regions on the earth would certainly scatter power in such a manner as to produce a moon echo much greater than $50 \mathrm{~km}$ deep. Calm sea or dry sandy desert without rocks may just be capable of producing a short enough echo. Trials with high-flying aircraft over different terrains are suggested.

Roberts: What is known from optical observations about the slope of mountains on the moon?

Gold: The " average" slope of the surface depends on the scale of irregularity in which we are interested. At the optically observable scale of one km a small per cent-perhaps five per cent-of the surface is inclined at angles greater than five degrees to the horizontal. It would be most interesting to pursue radar observations to one $\mathrm{cm}$ wavelength or shorter until the changeover to the rough surface seen optically has set in.

Yaplee: My measurements at $10,000 \mathrm{Mc} / \mathrm{s}$ over dry, sandy terrain show that there is a reduction of $15 \mathrm{db}$ in the power reflected at 10 degrees to the normal over that reflected at 5 degrees (C. R. Grant and B. S. Yaplee, Proc. I.R.E. 45, 976, 1957). 